\title{
Accurate Simulation of Mechanical Stresses in Silicon During Thermal Oxidation
}

\author{
A. Poncet \\ CNET-CNS, France Telecom \\ Chemin du Vieux Chêne, BP 98, F-38243 Meylan Cédex, FRANCE
}

\begin{abstract}
The aim of this paper is to present viscoelastic models to accurately simulate mechanical stresses which result from volume expansion during thermal oxidation or temperature ramps in silicon technology. Comparisons are made with wafer curvature measurements and it is shown that mechanical stresses can explain the "anomalously" fast initial regime during dry oxidation, without involving any additional chemical mechanism.
\end{abstract}

\section{Introduction}

Numerical models for local thermal oxidation have been developed for a decade $[3,4]$ starting from the well known Deal\&Grove 1D model [1]. Even at a very early stage of development, these 2D models were successfully applied to advanced isolation techniques like Sealed Isolation LOCOS (SILO) process [6]. This process is nowadays a candidate to compete with Poly-buffered LOCOS (PBL) for quarter micron CMOS technology [12]. Simulations of PBL have been performed more recently, due to the complexity resulting from the polysilicon layer which bends while being oxidized $[10,11]$. The effects of mechanical stresses have been introduced first in [6] to explain short bird's beaks, and the expression of stress induced reductions on diffusivity, reaction rates and viscosity are nowadays classically taken from the works of Kao, Suturdja et al. $[8,9]$. However, some progress was still expected for the accurate modelling of the complete mechanical stresses in Silicon, i.e. not only the additional stresses which result from local 2D effects and which are classically used in the present stress-reduced oxidation models; it includes stresses which result, even in 1D, from the expansion of silicon to form new oxide.

The aim of this paper is to present viscoelastic models to simulate more accurately this volume expansion. This approach includes the modelling of thermal stresses to account for temperature ramps. Comparisons are made with experiments on wafer curvature [2,7], and it is finally confirmed that, as anticipated by Fargeix and Guibaudo [5], mechanical stresses which are present in the thermally grown dioxide layers even in planar structures can explain the "anomalously" fast initial regime during dry oxidation.

\section{A Comprehensive Model for Moving Oxide/Silicon Interfaces}

The standard algorithm for LOCOS simulation consists in solving first the diffusionreaction equation at each time step in order to compute oxidant concentration, next growing a new oxide layer without any material deformation, and finally solving some equations (elasticity, Stokes, or viscoelasticity) in the upper layers in order to express the mechanical reaction of the rigid substrate by means of imposed velocities on the $\mathrm{Si} / \mathrm{SiO} 2$ interface.

Such a procedure fails when the silicon itself bends during oxidation, like in PBL for instance. Moreover, mechanical stresses computed by this way are uncomplete insofar as they 
represent only $2 \mathrm{D}$ effects in the overlayers. For instance, expressing sources of point defects versus stresses in silicon requires a complete stress computation. For these reasons, a new algorithm is proposed to deal with this paradox: to handle materials which can expand while they must keep uncompressible at given temperature and composition.

- the $\mathrm{Si} / \mathrm{SiO} 2$ interface is moved to express silicon consumption without any dilatation;

- next, viscoelasticity equations are provided with suitable extra terms to account for the expansion of the newly grown oxide;

- finally, the large tensile stresses which come out from a straighforward evaluation from velocities in the newly grown dioxide layer are updated, depending on what is needed:

* to get back usual profiles (extra 2D stress), stresses must return to zero in 1D areas;

* to get the exact stress tensor in all layers, and to update hydrostatic pressure (which is used in Uzawa algorithm to express uncompressibility), stresses which would result from unconstrained dilatation must be substracted; so doing, the lateral stresses in dioxide layers return to compressive as expected. This method has been validated first on thermal dilatation to check that, on one hand tangent stresses at the interface between two layers with different dilatation coeffficients are well balanced, and on the other hand that the volume expansion does not depend on boundary conditions: it must be for instance the same in a 1D structure with boundary conditions which impose displacements in only one direction, and a complete 2D structure; it is not the case with the "elastic" model which allows some compressibility of materials.

\section{Alternative models for mechanics}

Two models have been investigated: an incremental linear elastic one with maxwelian stress relaxation, and a complete elasto-visco-plasticone; stress-reduced viscosity from Suturdja \& Oldham [9] is available in both models. For sake of simplicity, the first model will be called "elastic", and the second one "viscous". Comparisons are shown on fig. 1.

With the "elastic" model, it is obviously impossible to decrease the magnitude of stresses in the newly formed oxide at each time step, because stress relaxation is applied only at the end of the time step; therefore, the stress dependent viscosity just leads to a stiffer stress reduction inside the oxide film, but this does not affect significantly stress in silicon.

On the other hand, as a linearisation loop is used in the "viscous" model, at least to express uncompressibility, the effect of reduced viscosity is observed all through the film thickness. In that case, mechanical stresses in silicon dioxide along the silicon/oxide interface are reduced accordingly.

However, in both cases the stress reduction is far from what is needed to decrease the wafer curvature significantly (fig. 2); this is in contradiction with a recent paper [14]. In the present model, the only way to get realistic curvatures is to put an anisotropy factor in the dilatation term: with $100 \%$ anisotropy, mechanical stresses reduce to the extra 2D stresses, as defined above.

Fig.1 - Stress relaxation in the SiO2 layer of a $I D$ structure for 3 different temperatures
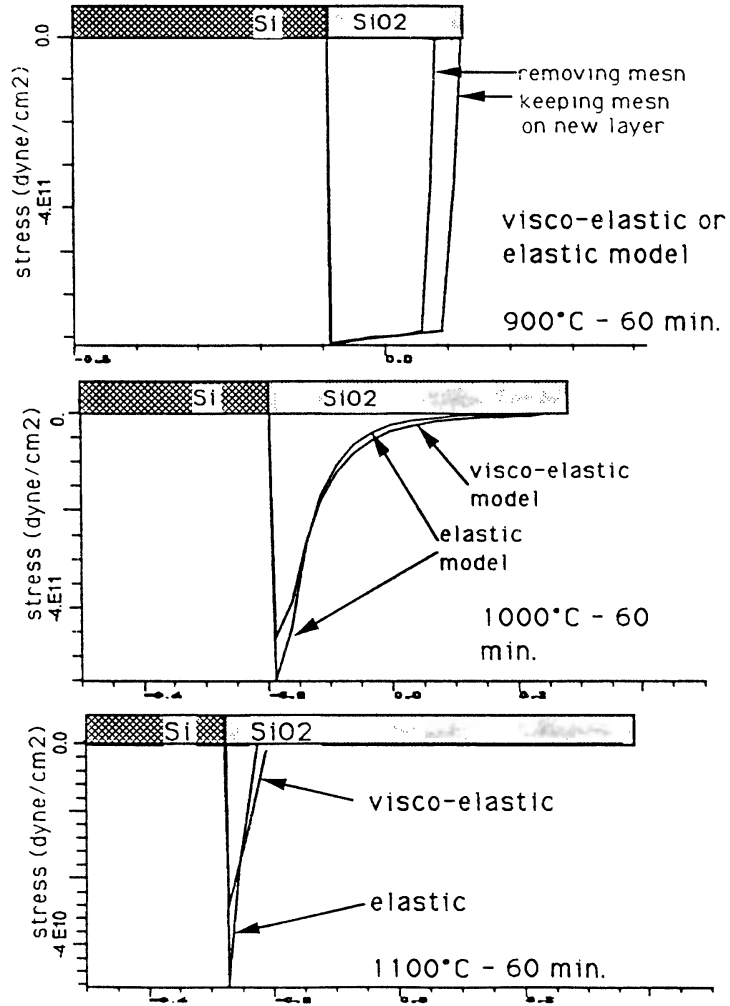
Unfortunately, the anisotropy factor, which is always greater than $90 \%$, exhibits a very strong temperature dependence which is still to be fitted with a wider range of experiments.

\section{Stress computation in full wafer oxidation}

The most usefull results published up to now in the literature to validate stress computations during thermal oxidation remain data on wafer curvature from Eernisse [2] because stress relaxation and stress dependent viscosity lead to stiff variation of mechanical stresses in the oxide film, and then to large uncertaincies when simulated stresses are compared with mean stress values found in some publications. On the other hand, experiments on microRaman analysis $[11,13]$ still suffer from a lack of accuracy to investigate local effects below 0.1 micron.

Curvature radii reported by Eernisse are large compared with the size of the area to simulate which, in turn, is large compared with the oxide film thickness (typical orders of magnitude are respectively $10^{6}, 10^{2}$ and $10^{-1}$ micrometers). While the behaviour of the "elastic" model is numerically stable, the "viscous" model exhibits large round off errors (fig. 2). In order to overcome this difficulty, a scaling has been designed: the curvature radius and the tangent component of stress in silicon along the silicon / silicon dioxide interface have been plotted versus wafer thickness. Two fair linear relationships versus the substrate thickness have been observed for the square root of the curvature radius and for the inverse of the tangent stress in silicon along the oxide intreface, provided that the dimension of the simulated area is large compared with this thickness (at least a factor of 5), and that the mesh is fine enough (fig. 3). This allows the dimensions of the simulated zone to be drastically reduced, and then curvature radii to be larger and less sensitive to round-off errors. As a consequence, mechanical stresses deduced from such simulations are more reliable as well.

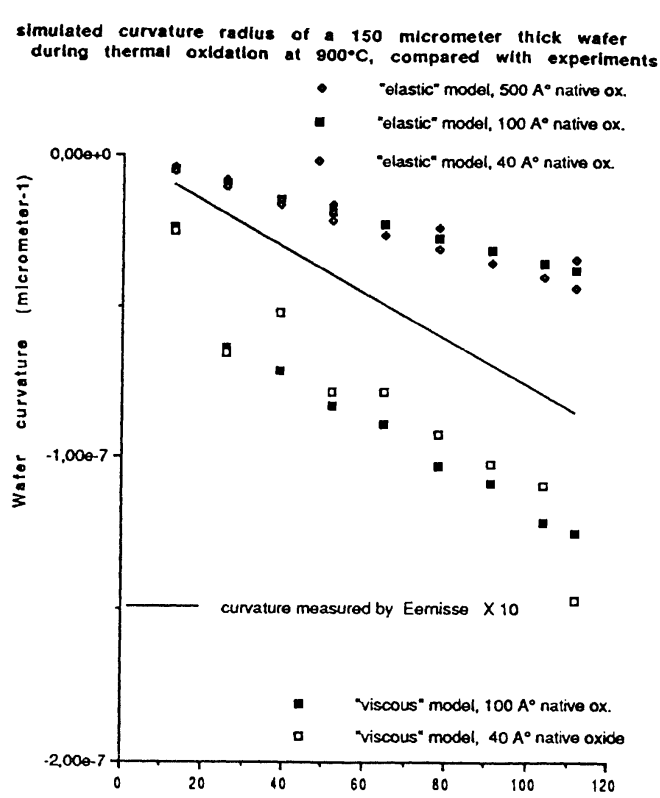

Fig. 2 - comparison between curvature from Eernisse and simulated curvatures using two alternative models

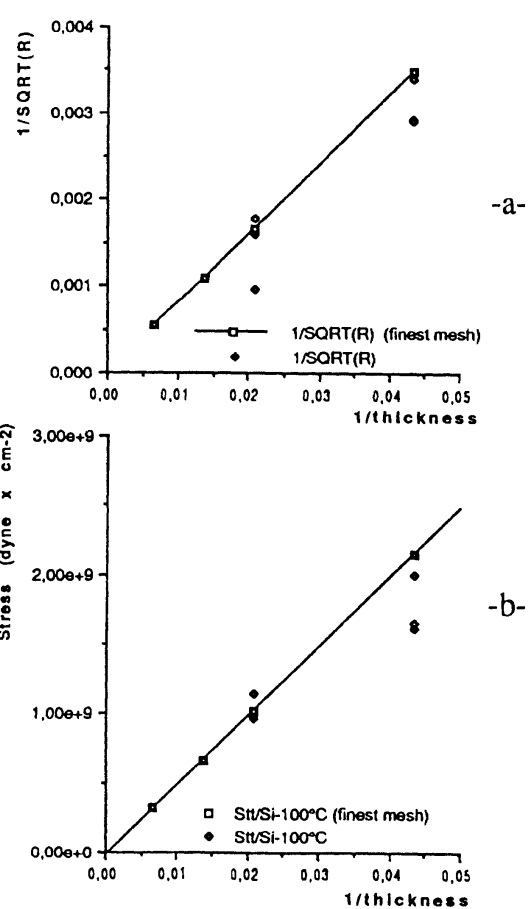

Fig. 3 - curvature - $^{1 / 2}(a)$ and tangent stress in Si along SiO2 interface (b) versus inverse substrate thickness. 


\section{Application to the early regime in dry oxidation}

As recommended by several authors, the best way to exhibit the fast initial regime during dry oxidation is to plot the inverse of oxide growth velocity versus oxide thickness; comparison between figs. 4-a and 4-b plots illustrate this recommendation. It is shown on fig. 4-b how this fast initial regime can be explained by using only stress reduced parameters without adding new physical or chemical mechanisms (like diffusion of two oxidizing species).

As mentionned above, total stresses are overestimated by more than one order of magnitude in the "elastic" model with isotropic dilation forces; let notice that this model is valid at temperature used in the simulations reported on fig. $2\left(900^{\circ} \mathrm{C}\right)$; therefore the activation volume to use in order to account for stress reduced diffusivity must be reduced by the same factor; for this reason, numerical results depicted on fig. 4 were obtained with an activation volume of $0.5 \AA^{3}$ More accurate stress profiles at $\mathrm{Si} / \mathrm{SiO} 2$ interface are necessary to obtain quantitatively good results with standard values of activation volumes (around $50 \AA^{3}$ ).
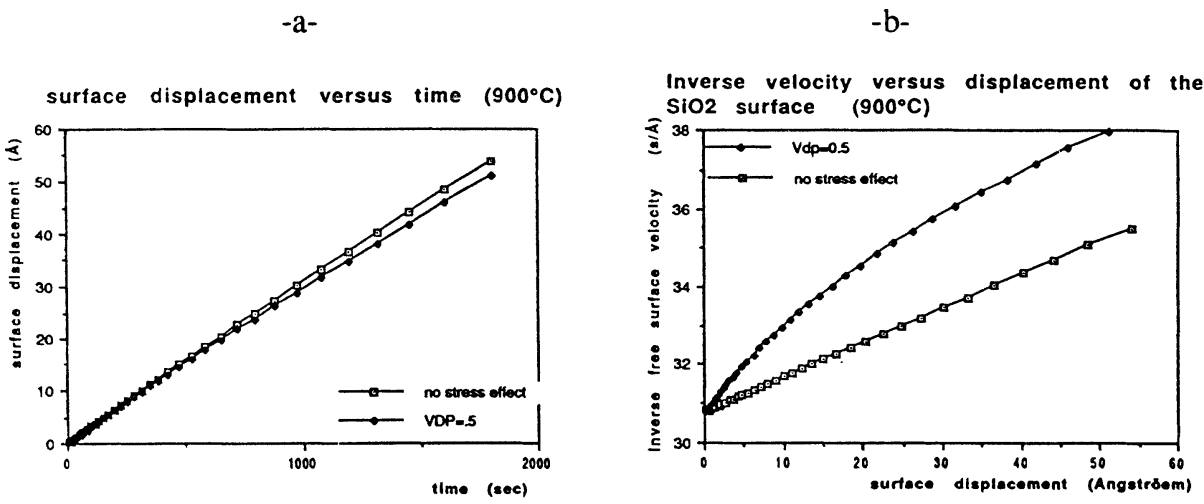

Fig. 4 - fast initial regime during dry oxidation explained by stress effect

\section{Acknowledgements}

This work has been partially supported by the European community under ESPRIT regulation. Projects $n^{\circ} 2197$ (STORM) and 7236 (ADEQUAT). Many thanks to S. K. Jones from GEC for his participation to the validation of models presented here and related software

\section{References}

[1] B.E. Deal, A.S. Grove "Genaral relationship for the thermal oxidation of silicon" J. Appl. Phys., 36 (13), 1965

[2] E. P. Eernisse "Stress in theremal SiO2 dring growth" Appl. Phys. Letter 35 (1) 1 July 1979

[3] A. Poncet "Numerical Simulation of Local Oxidation of Silicon" Summer Course IMEC Leuven , June 1983

[4] D.J. Chin, S.-Y. Oh, and R.W. Dutton "A General Solution Method for Two-Dimensional Non Planar Oxidation" IEEE/ED, Vol. ED-30 No. 9. Sept. 1983

[5] A. Fargeix and G. Guibaudo "Dry oxidation of silicon: A new model of growth including relaxation of stress by viscous flow" J. Appl Phys. 54 (12) Dec. 1983

[6] A. Poncet "Numerical Simulation of Local Oxidation of Silicon" IEEE/CAD, Vol. 4, No. 1, Jan. 1985

[7] B. Leroy "Stress and silicon insterstitials during the oxidation of a silicon substrate" Phil. Mag. B 55 (2) 1987

[8] D.-B. Kao, J. P. McVittie, W. D.Nix and K.C. Saraswat" Two-dimensional Thermal Oxidation of Silicon -II. Modelling Stress Effects in Wet Oxides" IEEE/ED Vol. ED-35, No. 1, Jan. 1988

[9] P. Suturdja and W.G. Oldham. IEEE/ED, Vol ED-36, No. 11, Nov. 1989

[10] A. Poncet "Numerical simulation of advanced isolation techniques" ICM'91 , Cairo, Dec. 1991

[11] I. De Wolf, J. Vanhellement, A. Romano-Rodriguez, H. Norström and H.E. Maes "Micro-Raman study of stress distribution in local isolation structures and correlatation with transmission electron microscopy, J. ppl. Phys. 71 (2), 15 Jan. 1992

[12] S. Deleonibus "A GIGABIT scalable SILO field isolation using Rapid Thermal Nitridation (RTN) of silicon" ESSDERC' 92 Conf. Leuven, Sept. 1992

[13] S. K. Jones, P.J. Pearson, C. Hill and A.V. Hetherington - STORM Internal report August 1992

[14] T. Uchida, N. Kotani and N. Tsubouchi "Verification of the Viscoelastic Oxidation Model Using Simple Test Structures" VPAD Conf. Proc. May 1993 